\title{
Indistinguishable Photons from a Single Molecule under Pulsed Excitation
}

\author{
Pietro Lombardi ${ }^{1,2, *}$, Maja Colautti ${ }^{1,2}$, Rocco Duquennoy ${ }^{3}$, Ghulam Murtaza ${ }^{1,2}$, Prosenjit Majumder ${ }^{1,2}$, and Costanza \\ Toninelli ${ }^{1,2, * *}$ \\ ${ }^{1}$ National Institute of Optics (CNR-INO), Largo Enrico Fermi 6, Firenze 50125, Italy \\ ${ }^{2}$ European Laboratory for Non-Linear Spectroscopy (LENS), Via Nello Carrara 1, Sesto F.no 50019, Italy \\ ${ }^{3}$ Dipartimento di Fisica e Astronomia, Universita degli Studi di Firenze, Via G. Sansone 1, Sesto F.no 50019, Italy
}

\begin{abstract}
Quantum light sources are crucial for the future of quantum photonic technologies and, among them, single photons on-demand are key resources in quantum communications and information processing. Ideal quantum emitters providing indistinguishable photons in a clocked manner, negligible decoherence and spectral diffusion, and with potential for scalability are today still a major challenge. We report on photostable and indistinguishable single photon emission from dibenzoterrylene molecules isolated in anthracene nanocrystals (DBT:Ac NCs) at 3K. The visibility of two-photon interference is preserved even when they are separated more than thirty times the excited-state lifetime, or ten fluorescence cycles. One of the advantages of organic molecules is the low-cost mass production of nominally identical emitters, that also allow for on-chip integration. These aspects combined with high spectral stability and coherence make them promising for applications and future quantum technologies.
\end{abstract}

\section{Introduction}

The generation and control of optical qubits has paramount importance for the realization of quantum communication[1] and computing protocols[2]. Cyber-security in our information-driven society is currently based on the assumption of the technical difficulty in factorizing large integers and will crumble after the construction of quantum computers. Single photons are natural carrier[3] of data in both free space and guiding media. In the quantum-protected version, two communication parties share entanglement or secret keys in the form of qubits which cannot be cloned by any eavesdropper[4]. In the past decades different quantum emitters such as quantum dots[5] and colour centers in diamond[6] were investigated for the generation of single photon qubits. Among them, individual Dibenzoterrylene molecules in a host anthracene crystal show promising performances both at cryogenic[7] [8] and room temperature[9]. In this contribution we fully characterize the emission from DBT:Ac NCs, in terms of the emitted photon coherence properties. In particular, two particle Interference (TPI), observed in the Hong-Ou-Mandel (HOM) effect, is analysed in order to determine the photon wave-packet overlap, required to realize all-optical gates and many communication protocols. We demonstrate high and stable visibility of twophoton interference in the HOM configuration, both under $\mathrm{CW}$ pumping and upon triggered excitation[10].

\section{Experimental Setup and Result Analysis}

Single molecules of Dibenzoterrylene (DBT) are an interesting choice for single photon emitters. DBT belongs to polyaromatic hydrocarbons (PAH). Embedded in small anthrecene crystals[11], they exhibit near-unity quantum yield,

\footnotetext{
*e-mail: pietroernesto.lombardi@ino.cnr.i

**e-mail: toninelli@lens.unifi.it
}

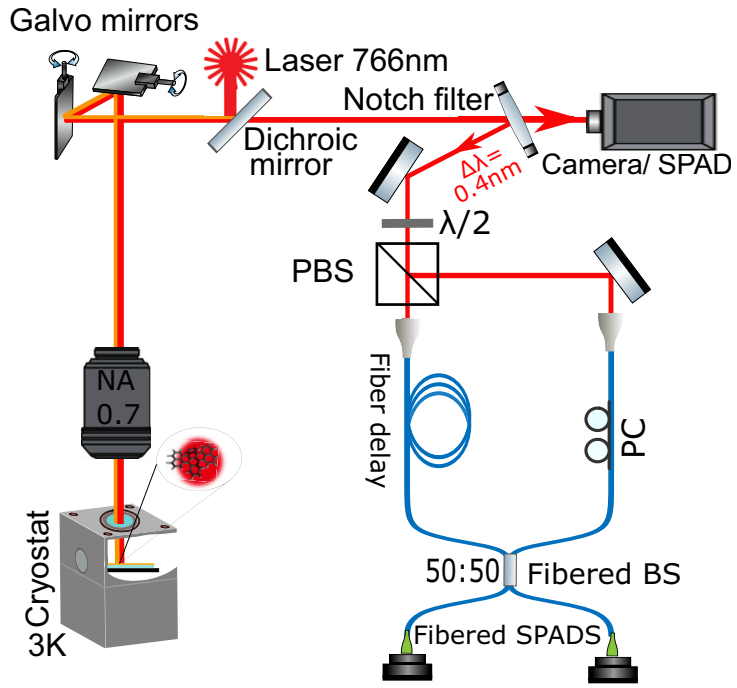

Figure 1. Experimental Setup: A Picoquant laser is used, which can be operated both in pulsed and CW mode. DBT:Ac NCs are kept inside a cryostat at $3 \mathrm{~K}$. An epi-fluorescence microscope with 0.7-N.A. objective is employed for excitation and emission collection. Notch Filter with 0.4-nm window filters the ZPL from the phonon side band (PSB). Different possible delay lines and a fibered beam splitter (BS) are used in the interferometer. EMCCD Camera and a free-space-coupled single photon avalanche diode (SPAD) are used to anaylize the ZPL and the PSB, whereas Fibered SPADs and a time to digital converter allow for reconstructing the histogram of the relative photon arrival times.

neglible blinking and Fourier-limited zero phonon lines (ZPLs) in the near infrared at low temperature. DBT:AC nanocrystals are placed inside a Cryostat working at $3 \mathrm{~K}$. Cryogenically cooled NCs are excited with tunable CW lasers or pulsed laser with adjustable repetition rate operating at a central wavelength of $766 \mathrm{~nm}$, with fluorescence collection of the ZPL at $783.5 \mathrm{~nm}$. The experimental setup is shown in Fig.1. Fluorescence light is collected with a $0.7-$ 
a

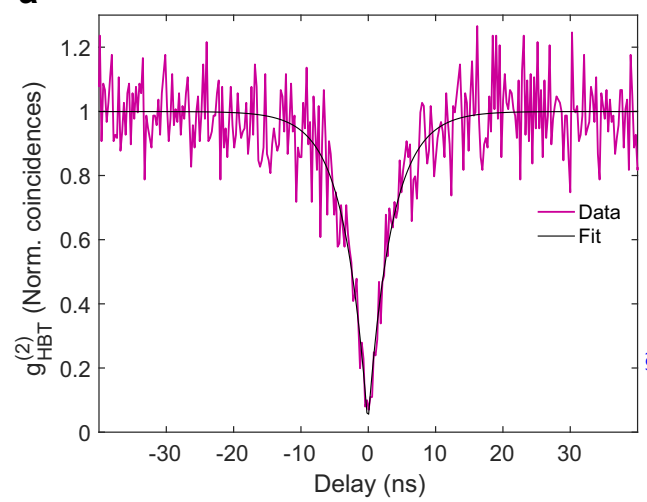

C

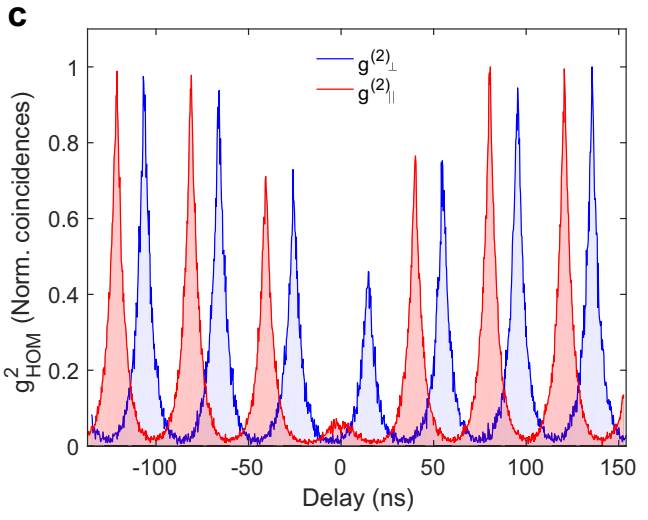

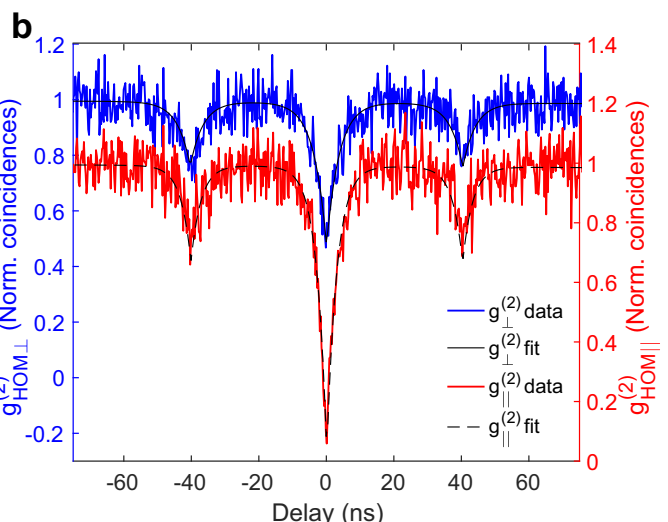

d

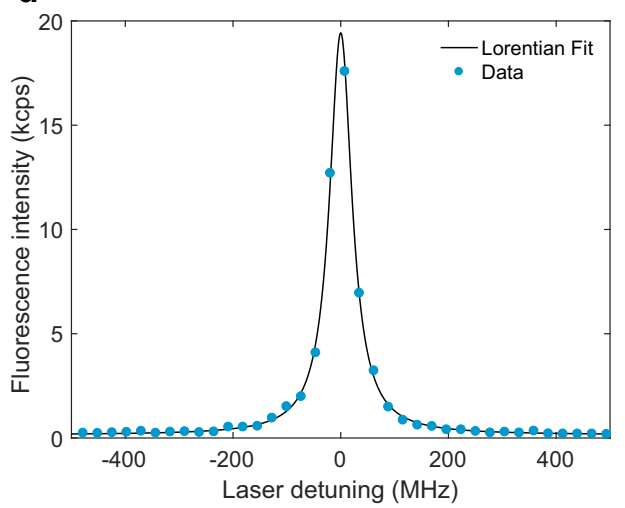

Figure 2. Optical characterization of molecular emission (a)Normalized second-order autocorrelation function of photon coincidences at both Fibered SPADS in continuous wave pumping $(b, c)$ Hong-ou-Mandel Experiment: Histogram of the relative photon arrival time for parallel (red) and orthogonal (blue) polarizations in (b) $\mathrm{CW}$ and (c) pulsed excitation. (d) Linewidth measurement by scanning resonant laser across ZPL, exhibiting a lorentzian profile with $F W H M=55.1 \pm 0.5 \mathrm{MHz}$.

Numerical-Aperture objective and isolated from the phonon sideband (PSB) with a 0.4-nm-wide notch filter in reflection. A 50/50 polarizing beam splitter separates the stream of photons into two arms. A fiber delay line of different possible length is added in one arm, whereas a fiber polarization controller (FPC) is included in the other arm. In this way, two non-correlated single-photon streams with different arrival times are obtained. A fiber beam splitter is used to recombine the two paths and coincidences are measured between the two output ports. Fiber polarization controllers are used to set parallel or orthogonal polarization in the two arms (switching between indistinguishable and distinguishable photons, respectively). Two fiber-coupled Single Photon Avalanche diodes (Fiber-SPADs) are employed for detection and the time histogram of coincidences is recorded by means of a time-correlated single photon counting module. DBT molecules are characterized both under $\mathrm{CW}$ and pulsed operation. Pulsed excitation is needed to have photons on demand. This optical setup works as Hanbury-Brown and Twiss (HBT) configuration when one arm (delay line) is closed. In Fig.2(a), the second order correlation function measured for an isolated DBT molecule is reported, showing high purity, with $g^{(2)}(0)=0.03 \pm 0.02$. Instead, HOM interference measurement is shown in Fig. 2(b\&c) for both indistinguishable(red) and distinguishable(blue) cases. The obtained Visibility is $V_{C W}=\frac{g_{\perp}^{(2)}(0)-g_{\|}^{(2)}(0)}{g_{\perp}^{(2)}(0)}=0.89 \pm 0.05$ un- der CW excitation, with 10-min integration time and 250coincidence as normalisation factor. The visibility under pulsed excitation is is calculated as a function of the peak areas within $\Delta t=26 n s$ which encompasses $96 \%$ area of photon wave packet around zero time delay in parallel $\left(A_{\|}\right)$and in the orthogonal $\left(A_{\perp}\right)$ scenario as follows: $\frac{A_{\perp}-A_{\|}}{A_{\perp}}$. This yields to $V_{P}=0.78 \pm 0.04$ with an integration time of 45 min. The pulsed laser has a tuneable repetition rate, which is adjusted in order to match the period between successive emission with the delay line. The laser repetition rate is finally adjusted to $24.79 \mathrm{MHz}=1 / \Delta t(\Delta t=40.3 \pm 0.2)$, based on the delay estimation obtained from the HOM measurement in CW (shown in Fig. 2(b)). Importantly, the parameters extracted from the best fit to the experimental data are consistent with independent measurements. In particular, the linewidth is measured by collecting the fluoresence intensity in the PSB while scanning the resonant laser across ZPL, yielding a $F W H M=55.1 \pm 0.5 \mathrm{MHz}$ (see Fig.1(d)), while the excited-state lifetime is estimated from the fluorescence decay, yielding $\tau=4.01 \pm 0.01 n s$. Non-classical interference of photons separated by different fluorescence cycles is shown in Fig. 3. The interference of two photons separated by first fluorescence cycle is clearly visible in Fig.3(a) corresponding to a repetition rate of $24.79 \mathrm{MHz}$. Different laser repetition rates are required in order to obtain the temporal overlap of further photons. Quantum inference with photons arriving after the fifth and tenth fluorescence cycles are pre- 
a

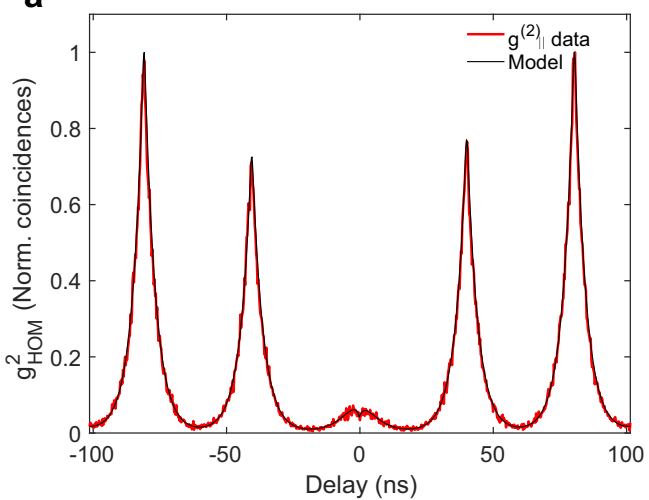

C

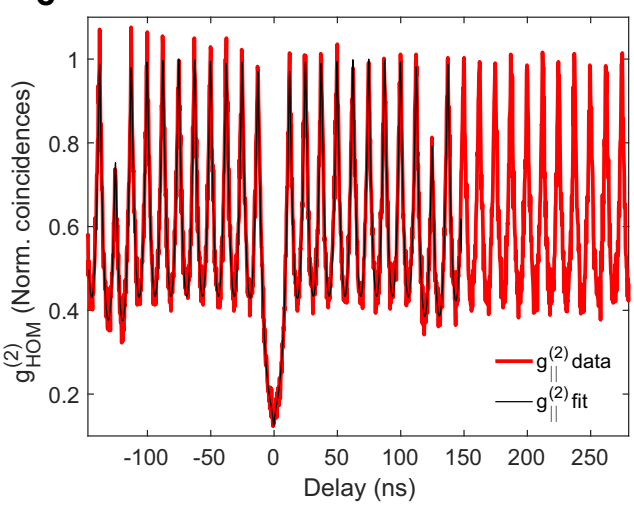

b

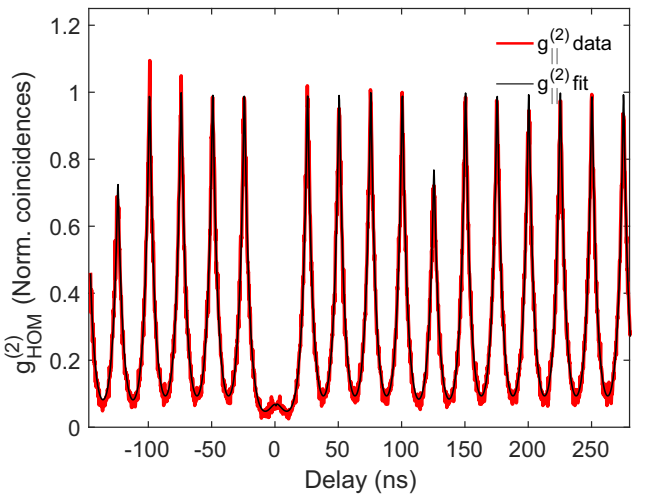

d

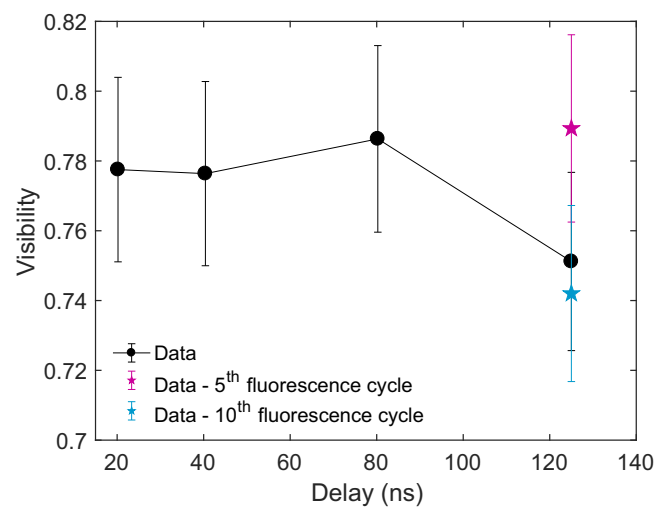

Figure 3. HOM Visibility: Quantum Interference Measurement of photons separated by (a) $1^{\text {st }}$ (b) $5^{\text {th }}$ (c) $10^{\text {th }}$ fluorescence cycles with $24.79 \mathrm{MHz}, 40 \mathrm{MHz}$ and $80 \mathrm{MHz}$ repetition rate, respectively. (d) HOM visibility as a function of Delay line with longest delay of $125 \mathrm{~ns}$ )

sented in Fig.3(b\&c), corresponding to $40 \mathrm{MHz}$ and $80 \mathrm{MHz}$ repetition rate, respectively. The longest delay time of $125 \mathrm{~ns}$ is achieved with a $25 \mathrm{~m}$-long fiber cable which is more than thirty times the excited state lifetime. Fig. 3(d) shows that visibility is only reduced with such long delay times. The overall visibility is limited by residual dephasing and could hence be increased to $96 \%$ by lowering the temperature to $1.5 \mathrm{~K}$. The finite extinction ratio of polarization optics, the spatial mode mismatch and PSB leaking through the notch filter are also accountable for lowering visibility. Overall spectral diffusion seems to be negligible, making this single photon source particularly interesting.

\section{Conclusion}

In conclusion, this paper reports indistinguishable single photons from an isolated single DBT molecule. This source also exhibits consistent HOM visibility for photon separated up to 30 times of excited state lifetime. Such type of molecular quantum emitters are easy to fabricate in hybrid system, have photostable emission and high degree of coherence.

\section{References}

[1] N. Gisin, R. Thew, Nature Photonics 1, 165 (2007)
[2] E. Knill, R. Laflamme, G.J. Milburn, Nature 409, 46 (2001)

[3] R. Uppu, F.T. Pedersen, et al, Science Advances 6, eabc8268 (2020)

[4] W.K. Wootters, W.H. Zurek, Nature 299, 802 (1982)

[5] X. Ding, Y. He, et al, Physical Review Letters 116, 020401 (2016)

[6] C. Kurtsiefer, S. Mayer, P. Zarda, H. Weinfurter, Physical Review Letters 85, 290 (2000)

[7] A.A.L. Nicolet, C. Hofmann, M.A. Kol'chenko, B. Kozankiewicz, M. Orrit, ChemPhysChem 8, 1215 (2007)

[8] J.B. Trebbia, P. Tamarat, B. Lounis, Phys. Rev. A 82, 063803 (2010)

[9] C. Toninelli, K. Early, J. Bremi, A. Renn, S. Götzinger, V. Sandoghdar, Optics Express 18, 6577 (2010)

[10] P. Lombardi, M. Colautti, R. Duquennoy, G. Murtaza, P. Majumder, C. Toninelli, Applied Physics Letters 118, 204002 (2021)

[11] S. Pazzagli, P. Lombardi, D. Martella, M. Colautti, B. Tiribilli, F.S. Cataliotti, C. Toninelli, ACS Nano 12, 4295 (2018) 